\title{
Reliability of Connections in Multilayer Networks under Shared Risk Groups and Costs Constraints
}

\author{
David Coudert, Florian Huc, Fabrice Peix, Marie-Emilie Voge \\ MASCotTe, InRIA-I3S(CNRS/UnSA), Sophia-Antipolis, France \\ \{firstname.lastname\}@sophia.inria.fr
}

\begin{abstract}
The notion of Shared Risk Resource Groups (SRRG) has been introduced to capture survivability issues when a set of resources may fail simultaneously. Applied to Wavelength Division Multiplexing Network (WDM), it expresses that some links and nodes may fail simultaneously. The reliability of a connection therefore depends on the number of SRRGs through which it is routed. Consequently, this number has to be minimized. This problem has been proved NP-complete and hard to approximate in general, even when routing a single request. Some heuristics using shortest paths have already been designed, however the cost (the usual routing cost, not in term of SRRG) was not part of the objective. In this paper we study the problem of minimizing a linear combination of the average number of SRRG per paths and the cost of the routing. The main result of our work is a column generation formulation that allows to solve efficiently the problem of maximizing the reliability of a set of connection requests in MPLS/WDM mesh networks with SRRGs while keeping the cost of the routing low.
\end{abstract}

Keywords: Reliability, Shared Risk Resource Groups, Integer Linear Programming, Column Generation.

\section{INTRODUCTION}

A challenging issue for service providers operating connection oriented networks, such as MPLS/WDM mesh networks, is to provide reliable routes for accepted requests. This has been fostered by the growing use of virtual and overlay networks which are embedded, in a transparent way, on an underlying network. Therefore, a great deal of researchers efforts have been concentrated on the design of efficient protection mechanisms at the backbone level (see for example [1]-[4]). At this level, the reliability of a single connection in the network might be measured as a function of the number of links it uses, each single link having its own probability of failure. However, in a physical network, several fibers, or links, may be packed together and hence physically cut simultaneously (earthquake, fire, malicious behaviour etc). Consequently, a single cause of breakdown induces several link failures. The concept of Shared Risk Resource Group (SRRG), or Shared Risk Group (SRG) for short, has been introduced to capture network survivability issues in this situation, i.e. when a set of resources may fail simultaneously. This concept can be extended to various kind of correlated failures.

A typical example of SRG is given by MPLS multilayer networks as described in [5]. This is illustrated by Fig. 1 where links $\mathrm{AH}$ and EI of the virtual network are both routed into the underlying network through link FG and so belong to a same SRG, the one associated to FG. Indeed a failure on the physical link FG induce the disruption of both virtual links AH and EI. In such multilayer networks with SRGs, the reliability of a connection is measured as a function of the SRGs through which it is routed.

Given a network and a set of requests, the problem of finding a routing minimizing the average number of SRG through which the route of a request goes has already been studied. It has been proved to be NP-hard [1], hard to approximate [6], and heuristic algorithms have been designed. For example [1] studied a tabu search heuristic algorithm for routing requests with different requirements. However, if we consider a network with edge cost, such an objective may lead to a costly routing. To avoid this, the objective to be minimized has to take into account two cost criteria. First we want to minimize the average failure probability, that is the average number of risks of failure on which a connection is dependent, as previously. Secondly, since an operator is interested in both the safety of the connections and their operating costs, the objective has to take into account the cost of the edges used along the route of the connections.

Hence, we concentrate on the problem of computing under capacity constraints a set of paths minimizing a linear combination of their edge costs and the number of SRGs through which they are routed. To our knowledge, this is the first time in the SRG context that such an objective, considering both the quality of the service offered to customers and the cost of the services, is proposed. Our main result is the first integer linear program (ILP) with column generation for the Minimum Average Color Flow problem, even when we consider only the objective of minimizing the average number of SRGs.

In this paper we also discuss a way to generate "realistic" virtual networks with SRRG. Since the simultaneous failures of links are correlated, a random assignment of SRRG to virtual links does not necessarily correspond to a feasible routing of the virtual topology into a real physical network, as was pointed out in [7]. The solution we propose avoid this problem and hence the experiments are run on graphs presenting more characteristics of real instances.

Our paper is organized as follows. In the next Section, we present the notations and network models used in the rest of the paper. Sec. III is dedicated to the Minimum Average Color Flow problem: once the problem is defined, we study its complexity and present two ILP formulations. In Sec. IV-A we remind some basics about column generation before applying it to Minimum Average Color Flow. We then detail in Sec. V the generation of our instances and the implementation of 


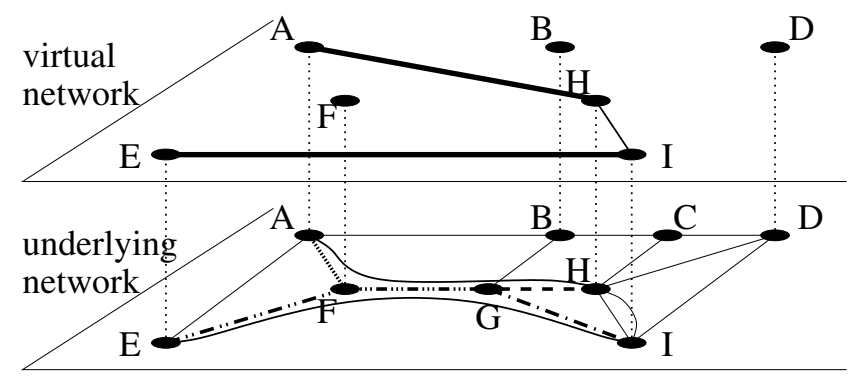

Fig. 1. Example of SRLG: virtual links AH and EI share the same risk FG.

our solving process, and finally we analyze the computational results before concluding.

\section{NeTWORK MODEL AND NOTATIONS}

The multilayer networks we consider are composed of two layers, a physical or underlying network and a virtual one.

The physical layer is modelled by a digraph $G_{\mathrm{PN}}=$ $\left(V_{\mathrm{PN}}, L_{\mathrm{PN}}\right)$, where $V_{\mathrm{PN}}$ is the set of nodes, and $L_{\mathrm{PN}}=$ $\left\{e_{1}, \ldots e_{m}\right\}$ is the set of links (i.e. unidirectional fibers). Similarly, the virtual network embedded in the physical one is modelled by the digraph $G_{\mathrm{VN}}=\left(V_{\mathrm{VN}}, L_{\mathrm{VN}}\right)$. Each arc $e \in L_{\mathrm{VN}}$ has a cost $\gamma_{e}$ per flow unit and a capacity $u_{e}$. The construction of the virtual networks used in our simulations will be described more precisely in Sec. V.

Each $\operatorname{arc} e_{i}$ of the physical network $G_{P N}$ gives in the virtual layer of a single risk group that we represent by a color $c_{i}$. More precisely, color $c_{i}$ is the subset of arcs of the virtual network routed through arc $e_{i}$ on the underlying layer, that is the set of arcs sharing the risk of failure of $\operatorname{arc} e_{i}$. The set $\mathcal{C}=\left\{c_{1}, \ldots, c_{m}\right\}$ is the set of colors (i.e. SRG) of the network. Note at that point that an arc of the virtual network may belong to several SRGs (= colors). However, following [1], [2], [5], we apply the simple transformation on $G_{\mathrm{VN}}$ described in [6], [8] : each multi-colored arc is replaced with a chain of monocolored arcs, so that each arc now belongs to exactly one color. For simplicity purpose we will denote also by $G_{\mathrm{VN}}$ the modified graph. Such graphs with SRG are refered to in the literature as colored graphs. Interested readers may refer to [9] for a model with multiple colors per edges, and to [8] for a preliminary comparison of both models.

Let $K$ be a set of connection requests over $G_{\mathrm{VN}}$, such that for each request $k \in K$, we are given its source $s_{k}$, its destination $t_{k}$ and its integral bandwidth requirement $d_{k}$. We assume that each request can be split into unitary requests to be served independently. Consequently, in the rest of the paper all the requests are unitary. For each request $k \in K, \mathcal{P}^{k}$ is the set of all the $s_{k} t_{k}$-directed paths in $G_{\mathrm{VN}}$ and $\mathcal{P}=\cup_{k \in K} \mathcal{P}^{k}$

Finally, we denote respectively by $\Gamma^{-}(v)$ and $\Gamma^{+}(v)$ the set of incoming and outgoing links at a node $v$.

\section{Minimum Average Color Flow Problem (MACF)}

\section{A. Problem definition}

An instance of the Minimum Average Color Flow problem consists in a digraph $G_{\mathrm{VN}}=\left(V_{\mathrm{VN}}, L_{\mathrm{VN}}\right)$, representing the virtual network, with arc cost $\gamma_{e}$ per flow unit and capacity $u_{e}$ for each arc $e \in L_{\mathrm{VN}}$. A set $\mathcal{C}=\left\{c_{1}, \ldots, c_{m}\right\}$ of colors -or SRG- partitioning the arc set $L_{\mathrm{VN}}$ is given, as well as a set of unitary connection requests $K$ and a real $\alpha \in[0,1]$.

A solution is a routing of all the requests satisfying all the capacity constraints.

The objective to be minimized is a convex combination of two cost criteria whose respective contributions can be adjusted through the parameter $\alpha \in[0,1]$. The first criterion, related to the operating cost, is the classical flow cost depending on the arc costs $\gamma_{e}$ divided by the total flow request $(=|K|)$. The second criterion, related to the safety of the connections, is the average number of colors - SRGs - to which belongs a path routing one unit of flow, that is the sum of the numbers of colors on which is routed each request divided by $|K|$. The objective function is then $\frac{\alpha}{|K|} \times($ classical flow cost $)+\frac{(1-\alpha)}{|K|} \times($ SRG flow cost $)$.

Note that when $\alpha=0$ the problem becomes the minimisation of the average number of SRG or in other word the average probability of failure for a connection, whereas if $\alpha=1$ the problem is reduced to a classical integral multicommodity flow.

\section{B. Complexity}

We now study the complexity of MACF under various hypothesis on the colored graph. We deduce these results for MACF from one of its subproblems: Minimum Color Path with Edge Costs problem (MCPwEC) which is the MACF problem with a single request, i.e. $|K|=1$, and unitary arc capacities. Thus, it consists in finding a path from a source $s$ to a sink $t$ minimizing a convex combination of the number of colors used by the path and of the classical path cost.

1) Null arc costs: With null arc costs, this problem is the Minimum Color Path problem (MCP) which has been proved NP-hard in [2] and hard to approximate within a factor $2^{\log ^{1-\delta}|\mathcal{C}|^{\frac{1}{2}}}$ in [6], with $\delta=\left(\log \log |\mathcal{C}|^{\frac{1}{2}}\right)^{-\varepsilon}$ and $\varepsilon<\frac{1}{2}$. These results were proved for undirected graphs, but their adaptation to the directed case is straightforward and induces the same factor for MACF.

2) Span 1: MCP can be solved in polynomial time when some conditions are satisfied on the span of the colors of the graph [6], [8], [10]. The span of a color is the number of connected components in the subgraph induced by the edges of that color. The influence of this parameter on the MCP complexity has been studied in [6], [11]. However, we show that the span has no such an influence on the hardness of MCPwEC.

Theorem 1: [6] When all colors have span 1, MCP is polynomial.

The proof is based on the fact that within a connected component of a color $c$, each vertex is reachable from any other vertex of the component using only edges of color $c$.

The span definition and Theorem 1 can easily be extended to the color symmetric digraph settings, i.e. when an arc and its reverse arc belong to the same color: the span is simply the 
number of strongly connected components and the property is still verified.

Let us now prove that the adjunction of edge costs deeply modify the complexity properties of MCP since MCPwEC is as hard to approximate as in the general directed case in the aforesaid symmetric settings.

Theorem 2: The MCPwEC problem on a color symmetric digraph is NP-hard and hard to approximate within a factor $2^{\log ^{1-\delta}|\mathcal{C}|^{\frac{1}{2}}}$ with $\delta=\left(\log \log |\mathcal{C}|^{\frac{1}{2}}\right)^{-\varepsilon}$ and $\varepsilon<\frac{1}{2}$ even when all colors have span 1 .

Proof: The proof is a reduction from MCP to MCPwEC. Let $G$ be a color symmetric digraph on color set $\mathcal{C}$ with unspecified span and two vertices $s$ and $t$ defining an instance of MCP. We construct an instance of MCPwEC on the digraph $G^{\prime}$ with convex coefficient $\alpha$ in the objective function. $G^{\prime}$ is the graph $G$ with two additional symmetric arcs of color $c$ and cost $X>\frac{1-\alpha}{\alpha}|\mathcal{C}|$ between each pair of strongly connected components of $c$. The cost of arcs from $G$ in $G^{\prime}$ is null and all the capacities are unitary.

An optimal st-path for the MCPwEC instance uses none of the added arcs since using only ones cost $\alpha X>(1-\alpha)|\mathcal{C}|$, which is greater than the cost of using the whole arc and color sets of $G$. Therefore, an optimal path in $G^{\prime}$ for MCPwEC is an optimal path in $G$ for MCP too. Indeed the two costs of a same path for MCPwEC and MCP differ only by the multiplicative constant $1-\alpha$. This last point concludes the proof.

From Theorem 2 we can deduce that in this special case MACF is as hard to approximate as its subproblem MCPwEC.

3) Color reduced to a single arc: Unlike MCPwEC and MCP, MACF remains NP-hard and hard to approximate when all colors are reduced to a single arc. Indeed in such a case, MCPwEC and MCP are merely polynomial shortest path problems while MACF still contains the Minimum Edge Cost Flow problem (MECF) as a subproblem. The MECF problem consists in routing a single integral request in a digraph with capacities and fixed arc costs, that is the cost of using an arc is the same whatever the flow value is on it, as long as it is positive. This problem is NP-hard and hard approximate within a factor $2^{\log ^{1-\varepsilon} n}, \forall \varepsilon>0$ [12].

Theorem 3: The MACF problem is hard to approximate within a factor $\approx 2^{\log ^{1-\varepsilon} n} \forall \varepsilon>0$ even when all colors are reduced to a single arc, unless $N P \subseteq D T I M E\left(n^{\text {polylog } n}\right)$.

\section{ILP Formulations}

In this section we present two ILP formulations for MACF based on the two classical multiflow formulations: the compact node-arc formulation and the exponential size arc-path one (see [13]). Although exponential, the arc-path formulation is more efficient than the compact one thanks to the possible use of column generation techniques.

1) Node-Arc Formulation: The binary flow variable $z_{e}^{k}$ for $e \in L_{\mathrm{VN}}$ and $k \in K$ gets value 1 if the virtual link $e$ is used to route the unitary request $k$ and 0 otherwise. The binary color variable $\chi_{k}^{c}$, for $k \in K$ and $c \in \mathcal{C}$, gets values 1 if the unitary request $k$ is routed through an arc belonging to the risk group represented by color $c$.

$$
\begin{array}{r}
\min \frac{1}{|K|} \sum_{k}\left(\alpha \sum_{c \in \mathcal{C}} \chi_{k}^{c}+(1-\alpha) \sum_{e \in A} \gamma_{e} z_{e}^{k}\right) \\
\sum_{e \in \Gamma^{+}(v)} z_{e}^{k}-\sum_{e \in \Gamma^{-}(v)} z_{e}^{k}=\left\{\begin{array}{r}
1 \text { if } v=s_{k} \\
-1 \text { if } v=t_{k} \\
0 \text { otherwise }
\end{array} \quad \forall k \in K\right. \\
\sum_{k \in K} z_{e}^{k} \leq u_{e} \\
z_{e}^{k} \leq \chi_{k}^{c(e)} \\
\quad \forall k, \forall e \in L_{\mathrm{VN}}
\end{array}
$$

Constraints (1) and (2) are the classical flow conservation and capacity constraints of a node-arc multiflow formulation. Constraint (3) ensures that whenever the flow for a request $k$ on an arc $e$ is positive, the color $c(e)$ of this arc is counted as used for this request, i.e. the value of $\chi_{k}^{c(e)}$ is forced to 1 , where $c(e)$ is the color of arc $e$. There are $|K|\left(\left|L_{\mathrm{VN}}\right|+|\mathcal{C}|\right.$ variables and $|K|\left(\left|V_{\mathrm{VN}}\right|+\left|L_{\mathrm{VN}}\right|\right)+\left|L_{\mathrm{VN}}\right|$ constraints.

The objective to be minimized is the average on all requests of a convex combination of the number of colors used by a request and of the classical cost of the route of a request.

2) Arc-Path Formulation: We now use binary variable $z_{p}^{k}$ which gets value 1 if the request $k \in K$ is routed on the path $p \in \mathcal{P}$, and 0 otherwise. As there might be a large number (exponential) of valid paths for each request, there might be a large number of variables in this family. As in the node-arc formulation, the binary variable $\chi_{k}^{c}$ has value 1 if the path used by request $k$ uses an arc of color $c$ and 0 otherwise.

$$
\begin{gathered}
\min \frac{1}{|K|} \sum_{k}\left(\alpha \sum_{c \in \mathcal{C}} \chi_{k}^{c(e)}+(1-\alpha) \sum_{e \in L_{\mathrm{VN}}} \sum_{P \in \mathcal{P}^{k} \mid e \in P} \gamma_{e} z_{P}^{k}\right) \\
\sum_{P \in \mathcal{P}^{k}} z_{P}^{k}=1 \quad \forall k \in K \\
\sum_{k} \sum_{P \in \mathcal{P}^{k} \mid e \in P} z_{P}^{k} \leq u_{e} \quad \forall e \in L_{\mathrm{VN}} \\
\sum_{P \in \mathcal{P}^{k} \mid e \in P} z_{P}^{k} \leq \chi_{k}^{c(e)} \quad \forall k \in K, \forall e \in L_{\mathrm{VN}}
\end{gathered}
$$

Constraints (4) ensure the satisfaction of all requests: for a given request $k$ there must be a path used to route it; we call $\sigma_{k}$ the associated dual variable. The capacity constraint (5) on each arc expresses that there can not be more paths using an $\operatorname{arc} e$ than there is bandwidth to fit them; we call $\omega_{e}$ the associated dual variables. As in the node-arc formulation, constraints (6) take into account the colors used to serve each request. The objective function is also similar. There are $|K|\left(\left|L_{\mathrm{VN}}\right|+1\right)+\left|L_{\mathrm{VN}}\right|$ constraints and empirically at most $10|K|$ variables, which is a lot smaller (for both variables and constraints) than in the node-arc formulation.

Note that to apply the column generation technique, or more precisely to define the auxiliary problem, we need the dual constraint associated to the large size family of variables $z_{p}^{k}$. 


\section{Solving Minimum Average Color Flow}

\section{A. Principle of column generation}

A column generation formulation is a transformation of a linear program containing a very large size variable family (e.g., exponential) into two subproblems: the master problem and the auxiliary problem. This decomposition relies on the Dantzig-Wolf decomposition [13]. It allows to consider only a subset of variables at a time.

Basically, starting with an initial reduced set of variables, we solve the master problem and obtain dual variable values that will be used by the auxiliary problem. Then, we solve the auxiliary problem to obtain new variables for the master problem, and so on. We repeat this loop until the auxiliary problem finds no more variables for the master problem. The solution of the master problem is then optimal (see [14]).

Column generation can be combined with branch-andbound techniques to solve integral linear programs with a large number of variables, see [15] for a nice overview.

\section{B. Column generation applied to MACF}

1) Master Problem: The master problem corresponds to the original arc-path formulation in which only a subset of the variables from the large size families $\left\{z_{p}^{k}\right\}_{p \in \mathcal{P}^{k}}$ is considered, all other variables of these families simply do not exist.

2) Auxiliary Problem: The auxiliary problem is deduced from the dual constraints of the master problem associated to the large size family of variable $z_{p}^{k}$ :

$$
\sigma_{k} \leq \sum_{e \in P}\left(\frac{(1-\alpha)}{|K|} \gamma_{e}+\omega_{e}+\pi_{e}^{k}\right) \quad \forall k, \forall P \in \mathcal{P}^{k}
$$

A solution of the auxiliary problem is a path $P$ violating this constraint. In our case the auxiliary problem is a shortest path problem on $G_{V N}$ with arc cost $\frac{(1-\alpha)}{|K|} \gamma_{e}+\omega_{e}+\pi_{e}^{k}$. We have to solve the auxiliary problem for each request $k$. Notice that the arc costs depend on the request, indeed the color cost $\left(\chi_{k}^{c(e)}\right)$ is not the same for each request.

3) Generation of the initial solution: The initial set of variables of the master program has to contain the non null variables of a feasible solution otherwise the column generation process can not start. To find this initial subset of variables we solve a classical minimum cost multiflow problem: from MACF we keep only the arc costs and capacities and we try to satisfy all the requests under the capacity constraints (see [13] for references on the minimum cost multiflow problem).

\section{Obtaining integral solutions: Branching}

We first solve with column generation a relaxation of the problem in which the variables $z_{P}^{k}$ and $\chi_{k}^{c}$ are fractional. Then we do branching to obtain an integral solution. During the branching a lot of computational time can be saved by considering the $\chi_{k}^{c}$ as fractional variables. Indeed, since all requests are unitary, once the flow variables are integral, the constraints on the $\chi_{k}^{c}$ variables force them to be integral too.

\section{EXPERIMENTS}

We have used CPLEX with a Concert Java interface and the open source MASCOPT library [16] to implement our model.

\section{A. Generation of instances}

To give a realistic significance to the colors, we assigned them to the virtual links according to a real routing of the virtual layer on a physical network as follows.

We used two networks as physical layers : NSFNET (14 nodes and 21 links) and BRAZIL (27 nodes and 70 links) [17]. Each physical link and node is associated a distinct color. if the network is already precolored with meaningful groups of risk, we may use them as well.

The virtual layer was generated using the following steps.

1) We generate an undirected graph on the physical vertex set using a pseudo random graph generator, implemented in MASCOPT, which accepts a set of constraints on the resulting graph. We choose the following ones: diameter at most 3 , minimum cut at least 2 , and number of links around 3 times the number of nodes.

2) We replace each edge by two symmetrical arcs.

3) We randomly generate unitary requests: 140 requests for NSFNET and 200 for BRAZIL.

4) We compute a double multiflow: the demands are routed on the generated graph whose arcs are themselves routed on the physical network simultaneously.

5) Each arc of the generated graph corresponds to a set of parallel virtual links : an arc is routed on a set of paths, each one giving a distinct virtual link. To each virtual link we assign the colors of the physical resources through which is routed its associated path.

6) The routing of the requests on the virtual network gives a weight to each virtual link. Thus, the capacity given to each virtual link $v_{l}$ is the minimum over each physical link $p_{l}$ of the proportion of utilisation of $p_{l}$ by $v_{l}$ among all virtual links multiplied by the capacity of $p_{l}$, that is: $\min _{p_{l} \ni v_{l}} \frac{\text { weight }\left(v_{l}\right) \text { capacity }\left(p_{l}\right)}{\sum_{v_{l}^{\prime} \in v_{p}} \operatorname{weight}\left(v_{l}^{\prime}\right)}$

7) We transform the networks given by the virtual links into a mono-colored graph [6] since at that point it may be multi-colored.

\section{B. Implementation}

We now briefly describe our implementation to allow a better understanding of next section V-C.

1) Columns generation: As explained in IV-A, the column generation is based on two problems (master and auxiliary). In our implementation both problems are solved using CPLEX. During the process, we only add columns to the master problem without removing any.

2) Obtaining an integral solution: We first implemented a branch-and-price algorithm using pseudo-cost to order the variables [15] and depth-first-search to visit the branch-andprice tree. However, our implementation is not yet efficient enough due to the lack of significant cuts. Therefore, we decided to obtain an integral solution via CPLEX using only the set of variables selected during the column generation process. The obtained solution might not be optimal, but close to it in our experimentation. We asked CPLEX to guarantee a gap with 

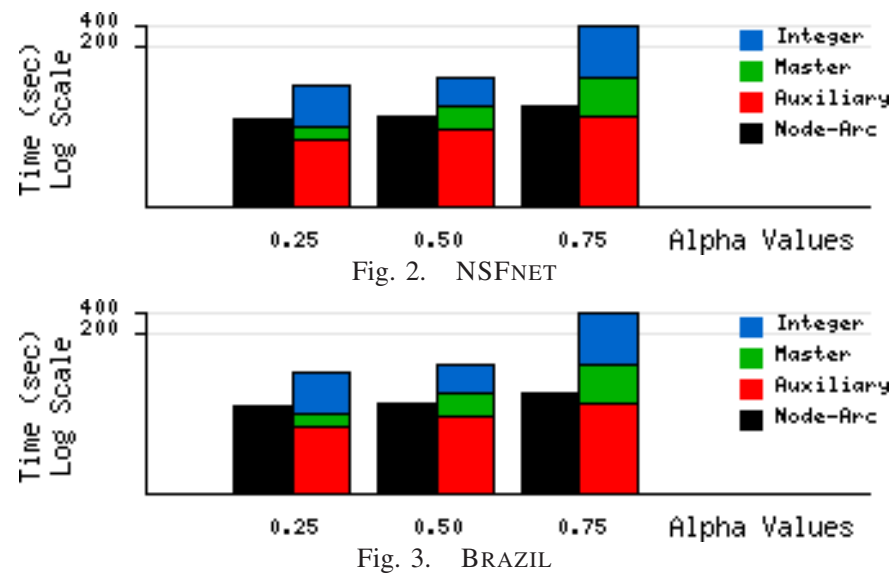

optimality of less than $5 \%$, and in practice the gap was less than $5 \%$.

\section{Computational results}

We made all our tests on a Intel Core $22.4 \mathrm{GHz}$ with $4 \mathrm{Mb}$ of L1 cache and $2 \mathrm{~Gb}$ of memory. We present our results in two graphics, one for NSFNET and one for BRAZIL. We solved each problem ten times using both formulations asking for an integer solution at at most $5 \%$ of the optimal (except when $\alpha=0.75$, where we ask for $10 \%$ ). The high of the rectangle represents the total time spent to find the solution. For the arc path formulation, the rectangle is splited accordingly to the time spent to solve the master problems, the auxiliary problems and to find an integral solution.

\section{1) MACF: column generation vs node-arc formulation:}

a) Computational time: Surprisingly, the node-arc formulation is always faster than the column generation formulation whereas there is a lot more variables in the first one. This can be partly explained by our implementation (we used a Java interface to call CPLEX which slows down the process) but also by the constraints on the color variables. Indeed in the node-arc formulation, a color constraint involves only two variables, while it may involve several ones in the column generation formulation. The color variables being the most numerous, it has a great impact on the resolution time.

In addition in the column generation formulation, each time we solve the master problem, we have to solve an auxiliary problem for each request (cf IV-B2).

b) Size of the instances solved: The column generation formulation allows to solve larger instances than the node-arc formulation, where the number of variables is too large.

c) Influence of the colors: The resolution time increases with $\alpha$ for both formulations due to the increasing importance of the color variables, which are 0-1 variables and numerous. It is worth noting that in the column generation formulation, the quality of the initial solution decreases when $\alpha$ increases since it does not take the colors into account at all.

\section{CONCLUSION}

In this paper we have investigated the problem of maximizing a linear combination of the reliability and the cost of a set of connection requests in mesh networks with SRRG. We have shown that MACF is NP-hard and hard to approximate, and we gave a column generation formulation to solve it allowing to obtain optimal or near optimal solutions in reasonable time.

We will now pursue our implementations and extend our work to the multiple-path problem where each connection request is protected by a SRRG-disjoint path and to other classical protection schemes. Note that SRRG-disjoint path in multilayer networks and with a different objective function currently under investigation in [18].

\section{ACKNOWLEDGMENTS}

This work has been partially supported by ANR JC OSERA, région PACA, European projects IST FET AEOLUS and COST 293 Graal.

\section{REFERENCES}

[1] L. Shen, X. Yang, and B. Ramamurthy, "Shared risk link group (SRLG)diverse path provisioning under hybrid service level agreements in wavelength-routed optical mesh networks," IEEE/ACM Transactions on Networking, vol. 13, no. 4, pp. 918-931, 2005.

[2] S. Yuan, S. Varma, and J. Jue, "Minimum-color path problems for reliability in mesh networks," in IEEE INFOCOM, vol. 4, 2005, pp. 2658-2669.

[3] C. Liu and L. Ruan, " $p$-cycle design in survivable WDM networks with shared risk link groups (SRLGs)," Photonic Network Communications, vol. 11, no. 3, pp. 301-311, May 2006.

[4] S. Ramamurthy and B. Mukherjee, "Survivable WDM mesh networks, part 1: Protection," in IEEE INFOCOM, 1999, pp. 744-751.

[5] D. Papadimitriou, F. Poppe, J. Jones, S. Venkatachalam, S. Dharanikota, R. Jain, R. Hartani, and D. Griffith, "Inference of shared risk link groups," IETF Draft, OIF Contribution, OIF 2001-066.

[6] D. Coudert, P. Datta, S. Perennes, H. Rivano, and M.-E. Voge, "Shared risk resource group: Complexity and approximability issues," Parallel Processing Letters, vol. 17, no. 2, pp. 169-184, Jun. 2007.

[7] D. Coudert, S. Perennes, H. Rivano, and M.-E. Voge, "Shared risk resource groups and colored graph: Polynomial cases and transformation issues," HAL, Tech. Rep. inria-00175143, Sep. 2007. [Online]. Available: http://hal.inria.fr/inria-00175143/fr/

[8] M.-E. Voge, "How to transform a multilayer network into a colored graph," in IEEE-LEOS ICTON/COST 293 GRAAL, vol. 3, Jun. 2006, pp. 116-119.

[9] A. Faragó, "A graph theoretic model for complex network failure scenarios," in Proceedings of the Eighth INFORMS Telecommunications Conference, Dallas, Texas, March 2006.

[10] P. Datta and A. Somani, "Diverse routing for shared risk resource groups (SRRG) failures in WDM optical networks," in IEEE BroadNets, Oct. 2004, pp. 120- 129.

[11] D. Coudert, S. Perennes, H. Rivano, and M.-E. Voge, "Shared risk resource groups and survivability in multilayer networks," in IEEELEOS ICTON / COST 293 GRAAL, vol. 3, Jun. 2006, pp. 235-238.

[12] G. Even, G. Kortsarz, and W. Slany, "On network design problems: fixed cost flows and the covering steiner problem," ACM Trans. Algorithms, vol. 1, no. 1, pp. 74-101, 2005.

[13] R. K. Ahuja, T. L. Magnanti, and J. B. Orlin, Network flows: theory, algorithms, and applications. Prentice Hall,, 1993.

[14] V. Chvatal, Linear Programming. Freeman, 1983.

[15] C. Barnhart, E. Johnson, G. Nemhauser, M. Savelsbergh, and P. Vance, "Branch-and-price: Column generation for solving huge integer programs," Operations Research, vol. 46, no. 3, pp. 316-329, 1998.

[16] J.-F. Lalande, M. Syska, and Y. Verhoeven, "Mascopt - a network optimization library: Graph manipulation," INRIA Sophia Antipolis, Tech. Rep. RT-0293, 2004. [Online]. Available: http://www-sop.inria. $\mathrm{fr} / \mathrm{mascotte} / \mathrm{mascopt}$

[17] T. Noronha and C. Ribeiro, "Routing and wavelength assignment by partition coloring," European Journal of Operational Research, vol. 1713, no. 3, pp. 797-810, Jun. 2006.

[18] S. Orlowski and M. Pioro, "Primal and dual formulations of network reconfiguration problems and related path generation issues," 2007, manuscript. 